\title{
Maintien et imposition de la paix en Somalie
} (Partie 2)

Jean-Paul Brodeur

\section{(2) OpenEdition \\ 12 Journals}

Édition électronique

URL : http://journals.openedition.org/conflits/688

DOI : $10.4000 /$ conflits.688

ISSN : $1777-5345$

Éditeur :

CCLS - Centre d'études sur les conflits lilberté et sécurité, L'Harmattan

Édition imprimée

Date de publication : 15 octobre 1998

ISSN : 1157-996X

Référence électronique

Jean-Paul Brodeur, " Maintien et imposition de la paix en Somalie (Partie 2) », Cultures \& Conflits [En

ligne], 29-30 | automne-hiver 1998, mis en ligne le 16 mars 2006, consulté le 30 mars 2021. URL:

http://journals.openedition.org/conflits/688 ; DOI : https://doi.org/10.4000/conflits.688

Ce document a été généré automatiquement le 30 mars 2021.

Creative Commons License 


\title{
Maintien et imposition de la paix en Somalie (Partie 2)
}

\author{
Jean-Paul Brodeur
}

1 Le mandat de l'UNOSOM II : son ambiguïté

2 Ce qu'il n'avait pu obtenir de Bush, Boutros-Ghali l'obtiendra de l'ONU. Le mandat de l'UNOSOM II incluait le désarmement systématique de toutes les factions, en plus de plusieurs autres tâches qui étaient du ressort du rétablissement des institutions somaliennes.

3 La principale ambiguité du mandat de l'UNOSOM II a été clairement énoncée dans le rapport de la CE-ONU. Dans son opuscule intitulé Un agenda pour la paix, Boutros Boutros-Ghali distingue entre divers concepts tels que la diplomatie préventive, le maintien (peace-keeping), le rétablissement (peace-making) et l'imposition de la paix (peace enforcement), et la reconstruction d'un pays (nation building). Le mandat de l'UNOSOM II comportait l'exercice de deux grandes prérogatives, soit le pouvoir d'instaurer un environnement sécuritaire (embargo sur les armes, escortes des convois humanitaires et surtout désarmement des factions) et celui de procéder à la reconstruction des institutions somaliennes. Or, d'après la commission d'enquête de l'ONU, seul le premier pouvoir était de la nature d'une imposition, alors que le second relevait exclusivement d'un pouvoir d'assistance ${ }^{1}$. " La résolution 814 du Conseil de sécurité invoque le chapitre VII de la Charte des Nations-Unies et confère des pouvoirs d'imposition à l'UNOSOM II. Ce faisant, elle distingue entre deux pouvoirs : le pouvoir de désarmer les milices somali, et le pouvoir d'assister le peuple somalien dans la restauration de la structure politique et des organes (gouvernementaux) de leur pays. L'UNOSOM II avait le pouvoir d'imposer le désarmement en usant de la force militaire d'après le chapitre VII de la Charte, quand la force était considérée comme nécessaire. Mais pour ce qui était du rétablissement de la structure politique du pays et de ses organes gouvernementaux, le rôle de l'UNOSOM II était limité à fournir de l'assistance plutôt qu'à imposer des solutions au peuple somalien²."

4 Le constat essentiel de CE-ONU (1994) est donc que UNOSOM II a confondu ces deux pouvoirs et que son action a consisté à tenter d'imposer aux Somaliens une 
reconstruction prédéterminée de leurs institutions politiques et gouvernementales, instituant ainsi une tutelle politique, que ceux-ci n'accepteront jamais, lui préférant la guerre. Un exemple de ce processus d'imposition de solutions politiques est la déclaration unilatérale de l'UNOSOM II que la loi qui serait appliquée en Somalie, serait le Code pénal somalien de 1962, qui datait d'avant le coup d'Etat militaire de 1969. l'UNOSOM II et la SNA, l'United Somali Congress (USC/SNA) étant explicitement mentionné dans la résolution. La résolution 837 fut initialement proposée par le Pakistan, dont vingt-quatre soldats avaient été tués le 5 juin 1993, et elle mentionnait le nom du général Aïdeed. Le nom de ce dernier fut retiré de la résolution à la suite des pressions américaines.

7 Cependant, le 17 juin suivant, le contingent marocain perdait plusieurs hommes lors d'une attaque de l'UNOSOM II contre l'enclave tenue par le général Aïdeed dans Mogadiscio-Sud. L'amiral américain Howe, qui était le Représentant spécial de Boutros Boutros-Ghali, se résolut alors à mettre la tête du général Aïdeed à prix pour $25000 \$$ US. Le 8 août, quatre soldats américains furent tués lorsque leur véhicule explosa sur une mine. Le Président Clinton ordonna que des commandos d'élite de la force Delta et des Rangers de l'Armée soient transportés d'urgence à Mogadiscio. Bien qu'ils fissent en théorie partie d'UNOSOM II, ils demeuraient sous commandement américain séparé et avaient pour mission de capturer Aïdeed et les cadres supérieurs de la SNA. Le 3 octobre 1993, au cours de l'opération la plus désastreuse de cette guerre, les forces d'élite américaines (Rangers et Delta Force) attaquèrent l'hôtel Olympia de Mogadiscio pour y capturer certains des principaux assistants de Aïdeed. Ils se retrouvèrent encerclés par les forces somaliennes et eurent 18 morts et 78 blessés. Le 6 octobre, le Président Clinton réunit ses principaux conseillers et ils conclurent qu'ils s'étaient trompés en suivant l'ONU dans sa tentative de " personnaliser le conflit " en mettant à prix la tête d'Aïdeed. Les Américains - et par la suite l'ONU - reconnurent que le général Aïdeed était un interlocuteur capital dans tout processus de rétablissement de la paix en Somalie ${ }^{3}$.

8 Ces péripéties ne sont pas seulement significatives des difficultés pour une coalition de suivre une politique cohérente et coordonnée. L'opération des troupes d'élite américaines contre Aïdeed constitue l'intrusion d'une action de police au sein d'une guerre néo-coloniale où les adversaires sont moins considérés comme des opposants qu'il faut vaincre que comme des délinquants qu'il faut soumettre à la justice.

UNOSOM I, UNITAF, UNOSOM II : la confusion des genres

10 Dans un article publié dans la revue Jeune Afrique, Géraldine Faes décrit en ces termes la confusion qui régnait en Somalie: " Peacemaking? Peacekeeping? Maintien de l'ordre ? Sécurité des convois? Désarmement? La plus grande confusion règne dans la tête des Somaliens comme, apparemment, dans celle des innombrables responsables, diplomates, fonctionnaires internationaux qui ont pris d'assaut les belles villas coloniales encore debout. Au Q.G. de l'UNOSOM (...) des expatriés s'affairent, montent des escaliers, rentrent dans un bureau pour en sortir aussitôt, tiennent des réunions de coordination du matin au soir. Personne n'a vraiment l'air de savoir qui est qui et qui fait quoi ."

Cultures \& Conflits, 29-30 | automne-hiver 1998 
11 D'une façon que nous estimons déterminante, la commission d'enquête établie par l'ONU confirme cette perception de chaos qui s'exprime dans l'article de Jeune Afrique. En effet, dans l'analyse qui clôt son rapport, la CE-ONU tente de répondre à la question suivante: " Pourquoi l'UNOSOM II a-t-elle mal jugé la situation? " (en se laissant entraîner dans une spirale de la violence). La réponse qu'apporte la commission est complexe. Celle-ci constate d'abord que UNOSOM II n'avait pas assez de conseillers civils d'expérience pour l'assister dans sa prise de décision ${ }^{4}$. En effet, les quelques conseillers politiques consultés ont considéré à tort que l'intervention des forces de UNOSOM II n'était qu'une affaire militaire ${ }^{5}$; quant aux militaires, ils n'avaient pas l'expérience requise pour bien jauger la situation ${ }^{6}$. Mais, peut-on se demander, de quelle expérience est-il question ici ? La réponse de la CE-ONU mérite d'être citée : " Faire la transition d'une posture de combat à une posture de maintien de la paix est en réalité très difficile pour un soldat professionnel. L'utilisation de la force pour atteindre son objectif est au coeur de la profession militaire. Dans le maintien de la paix, la force et l'imposition doivent être considérées comme le recours ultime après que tous les moyens pacifiques ont été épuisés. Il n'y avait personne pour enseigner les rudiments du maintien de la paix tant aux membres du quartier général de l'UNOSOM qu'aux contingents de soldats ${ }^{7}$."

Ce passage du rapport de la CE-ONU est capital : il nous apprend en effet qu'il faut faire une distinction absolument cruciale entre le maintien de la paix en son sens technique et étroit, et le maintien de la paix en son sens institutionnel et historique. Dans son sens technique, le maintien de la paix prend place à côté d'autres notions telles que le rétablissement et l'imposition de la paix et la reconstruction d'une nation; son sens est alors plus étroit et doit s'interpréter en fonction de chapitres déterminés de la Charte des Nations Unies, soit les chapitres VI et VII. Cependant, toutes les distinctions précédentes ne font sens pour la $\mathrm{CE}-\mathrm{ONU}$ que si elles sont subsumées sous le générique originel et plus compréhensible de " maintien de la paix ", tel qu'il a reçu son sens dans l'histoire de cette institution qui a pour nom les Nations Unies. Dans sa signification initiale, le " maintien de la paix " s'est défini comme le recours à la force ultima ratio, et il a continué d'en être ainsi par la suite. On pourrait, à cet égard, distinguer entre le concept opérationnel de maintien de la paix, qui prend place parmi d'autres notions présentes au sein de la Charte de l'ONU et du droit international, et le principe normatif $\mathrm{du}$ " maintien de la paix ", qui exprime l'exigence primordiale d'avoir recours à la force de façon ultime et minimale. Ce principe demeure toujours l'un des fondements de l'action de l'ONU.

13 Que le maintien de la paix ainsi entendu constitue effectivement l'un des fondements de l'action de l'ONU est bien ce que nous rappelle le rapport de la CE-ONU dans son antépénultième paragraphe: " Les Nations-Unies devraient se retenir d'entreprendre des actions futures d'imposition de la paix au sein des conflits internes des Etats. Si les Nations-Unies décidaient néanmoins d'entreprendre une opération d'imposition de la paix, son mandat devrait être limité à des objectifs spécifiques et l'usage de la force n'interviendrait que comme recours ultime, après que tous les remèdes pacifiques auraient été épuisés "8.

Jugement politique et néocolonialisme

Le présupposé de départ à toute opération de maintien de la paix est le consentement des autorités du pays ou du territoire impliqué à l'intervention de l'ONU ${ }^{9}$. Cette présupposition révèle un problème épineux. Il y a en effet deux cas distincts où la 
notion de consentement est problématique. Le premier de ces cas est simple : il n'y a effectivement pas consentement des autorités légitimes à l'intervention. Le second cas est plus épineux : les structures politiques d'un pays s'étant effondrées, il n'y a plus d'instances ou de personnes pour donner ou refuser leur consentement à une intervention. Ce cas est compliqué pour plusieurs raisons. D'abord, juger que les instances décisionnelles d'un pays se sont dissoutes constitue un acte qui peut comporter une part sérieuse d'ethnocentrisme. En effet, le jugement de l'observateur de l'ONU peut être moins attentif à la persistance dans un pays des structures traditionnelles de pouvoir qu'à l'érosion des structures politiques qui sont modelées sur celles de son propre pays. Ainsi, toute l'expérience tentée par le premier Représentant spécial du Secrétaire général de l'ONU, Mohamed Sahnoun, était précisément de résister à l'ethnocentrisme et de s'efforcer de réactiver les structures traditionnelles de décision du peuple somalien, alors que les structures calquées par Syad Barre sur celles des pays du Bloc de l'Est s'étaient, elles, complètement effondrées. Il est loin d'être sûr que cette décomposition d'une greffe postiche équivalait à l'effritement véritable de toute autorité légitime en Somalie.

Un second problème a été mis en lumière par la CE-ONU, qui était présidée par un magistrat de la Zambie : " La conclusion qu'un pays est sans gouvernement, comme elle fut formulée à l'égard de la Somalie par les Nations-Unies, a des conséquences légales et politiques si profondes que des critères mûrement délibérés semblent requis pour y parvenir. Si les Nations-Unies opèrent dans un pays qui a été ainsi caractérisé, elles doivent nécessairement accepter au moins quelques-unes des responsabilités d'Etat fondamentales qui sont la prérogative traditionnelle d'un gouvernement et cela soulève le fantôme d'une tutelle des Nations-Unies ou du néo-colonialisme "10.

Cette difficulté a été systématiquement - et très habilement - exploitée au cours de la crise somalienne par le général Aïdeed, qui s'est posé en champion de la souveraineté somalienne face à l'ingérence de l'ONU. maintien de la paix comme concept opérationnel. En tant que concept opérationnel, le maintien de la paix (peace-keeping) prend place à côté d'une kyrielle d'autres stratégies de pacification, que nous avons précédemment en partie énumérées ${ }^{11}$. Comme nous l'avons déjà constaté, les interventions en Somalie ont servi de banc d'essai à toutes ces nouvelles stratégies ${ }^{12}$. Il est toutefois possible que ce banc d'essai n'ait eu d' autre existence, que dans la tête des fonctionnaires de l'ONU et du gouvernement américain et que les subtiles différences entre le maintien, le rétablissement et l'imposition de la paix, sans compter " la reconstruction de l'Etat somalien ", n'aient pas résisté à l'épreuve du terrain. Pour le peuple somalien, les interventions de l'ONU ont dégénéré dans une agression progressivement caractérisée par sa seule violence. Nous tenterons maintenant d'examiner dans quelle mesure les règles qui devaient structurer l'action des troupes déployées en Somalie dans le cadre de l'UNOSOM I, l'UNITAF et l'UNOSOM II ont reflété dans leur formulation et dans leur application la différence entre ces trois interventions successives. Nous conduirons cet examen en rapport avec divers facteurs, et en examinant plus particulièrement une expérience précise, celle des troupes canadiennes.

Le choix des troupes 
21 Les troupes canadiennes qui devaient être déployées en Somalie ont été initialement choisies en fonction d'une participation au sein de l'UNOSOM I. Ces troupes appartenaient au Régiment aéroporté canadien. Ce régiment de parachutistes était formé des soldats canadiens les mieux entraînés et les plus motivés pour le combat. Cette unité, la plus agressive parmi les Forces armées canadiennes, était peut-être la moins appropriée à une opération de maintien de la paix, comme le démontre la recherche sur le déploiement de parachutistes dans le cadre d'opérations de maintien de la paix ${ }^{13}$. Les Canadiens ne furent pas les seuls à choisir ce genre d'unité, les Belges ayant également choisi leurs Para-commandos.

Dans le contexte actuel des restrictions budgétaires qui s'abattent sur les forces armées, les seules unités qui sont conservées pour un déploiement rapide à l'extérieur d'un pays sont des unités d'élite composées de parachutistes et de commandos. Seules ces unités sont disponibles pour une mission à l'étranger, ne serait-ce que dans le cadre d'une mission de maintien de la paix qui contredit, de leur propre aveu, leur raison d'être (le combat).

23 Mal choisie pour ce qui était de son agressivité dans le combat, cette unité de parachutistes possédait néanmoins les qualités professionnelles pour sa mission. Elle devait initialement être déployée au nord de la Somalie, près du port de Bossaso. Elle devait y édifier des barrages routiers et veiller à ce que l'aide humanitaire soit aiguillée sur les bonnes routes et protégée contre d'éventuels pillards opérant dans le port de Bossaso. Pour le dire plus simplement, cette unité devait initialement conduire ses opérations à pied. Or, sa mission changea de façon abrupte lorsqu'il fut décidé de l'intégrer à l'UNITAF. Non seulement elle serait déployée sur un terrain entièrement différent (le désert, au lieu d'une petite ville portuaire), mais surtout la tâche essentielle de ses membres consisterait à effectuer des patrouilles motorisées dans des véhicules blindés. Le temps manquant pour changer d'unité, on entraîna de façon précipitée les membres du Régiment aéroporté à devenir une unité motorisée. L'exigence de maintenir le moral du Régiment aéroporté qui s'entraînait depuis plus de deux ans pour des missions qui avaient avorté joua également un rôle prépondérant dans le choix de cette unité.

En d'autres termes, le changement de mission n'eut aucune répercussion sur le choix des troupes canadiennes qui seraient déployées en Somalie. Les troupes choisies pour l'UNOSOM I furent recyclées dans l'UNITAF, en dépit du changement de mission et de l'entraînement en porte-à-faux de ces troupes.

L'entraînement

En effet, l'entraînement des troupes canadiennes fut dicté par les exigences de la mission initiale à laquelle elles devaient participer, c'est-à-dire UNOSOM I. Les troupes canadiennes s'entraînèrent donc dans la perspective de leur déploiement au nord de la Somalie, dans une zone portuaire. C'est après que son entraînement eut été terminé que le régiment aéroporté canadien a donc appris qu'il serait déployé dans la région de Belet Huen, au centre désertique de la Somalie. On procéda hâtivement à un nouvel entraînement de ces fantassins aéroportés, pour les transformer en des troupes motorisées. A part ce changement dans des habilités professionnelles aussi élémentaires que nécessaires - la capacité de conduire un véhicule blindé -, le changement de mission de l'UNOSOM I (chapitre VI de la charte de l'ONU) à l'UNITAF (chapitre VII de cette même Charte) ne fut suivi d'aucune modification dans l'entraînement des soldats. Comparaissant devant deux commissions 
gouvernementales sur l'intervention du Canada en Somalie, des soldats canadiens ont répondu qu'ils considéraient l'entraînement qu'ils avaient reçu pour intervenir en Somalie comme nul et non avenu ${ }^{14}$. entraînement s'explique en grande partie par le fait que le Canada était soumis aux fluctuations abruptes de la politique américaine sur la Somalie. Ces fluctuations ont dicté les changements de mandat des troupes de l'ONU. Même rendus en Somalie, les Canadiens ne savaient pas où ils allaient être déployés: " Nous attendions un avion Hercule qui devait nous conduire à Belet Huen. Ce n'était pas notre destination première, mais les plans avaient été changés par les Américains, qui nous avaient attribué un nouveau secteur ${ }^{117}$.

Il est possible que les officiers aient été mieux informés que leurs hommes, mais seulement au plus haut niveau.

Le sort des Canadiens ne nous paraît toutefois d'aucune façon exceptionnel et il a été partagé, pour autant que nous puissions en juger, par les contingents fournis par les autres Etats membres de l'ONU.

Une opération de maintien de la paix donne lieu à l'énoncé de règles d'engagement (RDE) - rules of engagement (ROE) - et de procédures d'opération standardisées (POS) standard operational procedures (SOP) ${ }^{18}$. Ces règles régissent l'usage de la force militaire et de la coercition (par exemple, pour ce qui est de la coercition, une règle déterminera les conditions de détention des personnes détenues et les circonstances dans lesquelles elles peuvent l'être). L'importance de ces règles varie avec la probabilité qu'un contingent opérant dans le cadre d'une opération de maintien de la paix doive effectivement recourir à la force. Selon cette logique, la nécessité de posséder des RDE et des POS claires est devenue croissante dans la mesure où le passage de l'UNOSOM I à l'UNITAF et à l'UNOSOM II a représenté une progression dans l'éventualité du recours à la force armée. 

séquence graduée des réponses appropriées à une situation caractérisée (une menace et $d u$ harcèlement par une ou des personnes sans arme, une menace par une personne armée, une menace par des personnes se servant d'armes lourdes, une menace aérienne).

Ces règles appellent au moins deux remarques. D'abord, elles sont entièrement soustendues par le principe de l'autodéfense. Cette remarque prend tout son sens, quand on se rappelle que l'UNITAF fut une coalition formée en vertu du chapitre VII de la Charte de l'ONU, qui autorise de façon explicite le recours à la force. Quelle que fût la stratégie d'intervention choisie, il semble que le principe du maintien de la paix, avec son

Cultures \& Conflits, 29-30 | automne-hiver 1998 
corollaire de l'utilisation d'une force minimale, continue toujours d'opérer. Nous remarquerons en outre, qu'à l'exception d'une menace aérienne, la séquence graduée des réponses prévues fut toujours la même : l'avertissement verbal, la force minimale, les coups de feu d'avertissement et la force meurtrière ${ }^{21}$. Cette uniformité dans les réponses permises à l'agression n'est pas de nature à aider les troupes à pratiquer une approche différenciée sur le terrain.

La situation de l'UNOSOM II par rapport aux RDE et aux POS est confuse. En premier lieu, les auteurs du rapport de la CE-ONU ont souligné qu'à cause du manque de personnel d'expérience parmi les troupes de l'UNOSOM II, les troupes avaient été laissées sans POS et sans lignes directrices pour guider leur action (CE-ONU, 1994, par. 258). En second lieu, il est clair que les résolutions 814 et 837 impliquent un recours à la force de nature proactive : le désarmement systématique des belligérants, l'arrestation des responsables de la SNA et la chasse au général Aïdeed débordent largement le cadre de la simple réponse à une menace de circonstance. D'une manière similaire, le recours à des moyens comme des hélicoptères de combat et des bombardements aériens " de précision " implique d'emblée le recours à une force meurtrière qui éclate sans avertissement préalable. Les opérations conduites par les Rangers américains et les commandos de la Delta Force reposaient ainsi de façon essentielle sur la surprise et ne pouvaient par définition s'annoncer à l'avance par des avertissements. Il n'en reste pas moins, en troisième lieu, que la CE-ONU blâmera gravement l'UNOSOM II d'avoir manqué aux exigences fondamentales du maintien de la paix, entendues comme l'impératif de ne recourir à la force qu'en dernière instance. Nous reviendrons plus tard sur ces trois constatations qui sont, sous certains de leurs aspects, contradictoires. Par exemple, le recours proactif à la force ne se concilie pas d'emblée avec l'exigence de n'en faire usage qu'en dernier recours. Nous verrons maintenant brièvement les conséquences sur le terrain des nombreuses lacunes que nous avons relevées dans les RDE et les POS.

Les bavures de l'UNITAF

Comme nous l'avons rappelé, non seulement le Canada est perçu, avec la Norvège de Dag Hammarskjöld, comme le pays inventeur de l'idée de maintien de la paix par des troupes au service de l'ONU, mais des soldats canadiens ont participé à une exception près à toutes les opérations onusiennes de maintien de la paix. La participation du Canada à ce type d'opération a généralement été couronnée de succès, le soldat canadien étant souvent présenté dans la littérature sur le maintien de la paix comme l'un des modèles du Casque bleu ${ }^{22}$.

La fierté légitime des Canadiens, à l'égard de la participation des Forces armées canadiennes dans les opérations de maintien de la paix de l'ONU ${ }^{23}$, a été sérieusement mise à mal par le comportement des soldats canadiens en Somalie. Il a été établi qu'ils avaient battu pendant toute une nuit un adolescent somali du nom de Shidane Arone et que celui-ci était mort de ses blessures. Il existe en outre plusieurs allégations sérieuses à l'effet que des membres du contingent canadien en Somalie auraient tendu des embuscades à des pillards pour ensuite les exécuter sommairement et qu'ils se seraient livrés à d'autres violations graves des droits de la personne. En outre, le contingent canadien aurait compté des soldats affiliés à des groupes racistes de l'extrême-droite. $\mathrm{Au}$ moment où nous rédigeons cette étude, plusieurs soldats canadiens déployés en Somalie ont été condamnés par des cours martiales; le régiment aéroporté canadien a été dissous; enfin, le comportement des troupes canadiennes en Somalie et des 
fonctionnaires du ministère canadien de la Défense impliqués dans cette opération fait maintenant l'objet des travaux d'une Commission royale d'enquête, dont les audiences publiques font quotidiennement la manchette des journaux canadiens.

Les troupes canadiennes ne sont pas les seules à s'être rendues coupables de grossiers abus de pouvoir lors de leur déploiement en Somalie. Plusieurs soldats du contingent belge ont également été traduits en cour martiale. L'organisme African Rights a produit un rapport très sévère sur la conduite des troupes belges lors de leur participation dans l'UNITAF et surtout de leurs opérations dans le cadre de l'UNOSOM II. Les allégations d'inconduite contre d'autres membres de l'UNITAF sont nombreuses, ceux-ci étant décrits comme des soldats ivres se livrant à des actes incontrôlés d'agression L'explication de ces abus excède le cadre de cette étude. Nous nous contenterons de noter qu'il existe peu de parallèles au sein des interventions de l'ONU pour le maintien de la paix avec les ratages de la Somalie. Quelles que soient les raisons que l'on alléguera pour les difficultés de cette opération, le caractère improvisé et même à certains égards inexistant du cadre normatif qui devait régir l'intervention devra figurer en bonne place dans l'explication proposée. Pour l'essentiel, on s'en remit à la tradition mise en place par les interventions précédentes de l'ONU. Ce que révèle audelà de toute contestation le désastre somalien est précisément le caractère obsolète et dysfonctionnel des normes développées " sur le tas " dans les opérations précédentes et la nécessité de procéder à une remise en cause fondamentale du cadre imprécis des opérations de maintien de la paix de l'ONU.

Dans le quatrième appendice de son rapport, la CE-ONU a élaboré une liste des incidents violents qui se sont produits en Somalie entre le 5 juin et le 22 octobre 1993. Cette période correspond en gros à la " guerre " à laquelle les forces de l'ONUSOM et celles de la SNA du général Aïdeed se sont livrées lors de l'intervention des forces de l'ONU en Somalie. Cette liste est incontestablement incomplète et fut dressée pour l'essentiel à partir des événements qui sont arrivés dans la région de Mogadiscio. En dépit toutefois de ses lacunes, cette liste est éclairante. Nous en avons fait une analyse sommaire, comptant les incidents et les regroupant en catégories. Voici les résultats de cette analyse.

La liste élaborée par la CE-ONU comporte deux colonnes d'incidents. Dans la colonne de droite se trouvent les actions hostiles de la SNA contre l'UNOSOM ; on trouve dans la colonne de gauche les actions violentes de l'UNOSOM. Le nombre des agressions de la SNA contre les forces de l'UNOSOM se chiffre à 140 . Voici comment se répartissent ces actions $^{24}$ :

Tableau 1 : actions de la SNA contre les forces de l'UNOSOM 
Tireur d'élite (sniper) 1

Tirs d'artillerie 1

Tirs de mortier 80

Tirs de RPG 25

Plus de la moitié de ces actions consistent en des tirs de mortier (80), auxquels s'ajoutent les tirs de RPG (27). Dans leur très grande majorité, ces tirs ont été effectués contre quatre cibles : l'aéroport de Mogadiscio, l'ambassade des Etats-Unis, le New Port et la base Sword.

Voici maintenant le tableau des actions des forces de l'UNOSOM contre celles de la SNA. Bien que la plus grande partie de leurs troupes aient quitté la Somalie en mai 1993, à la fin de l'opération " Rendre l'espoir " (UNITAF), les Etats-Unis fournirent une force d'intervention rapide (Quick Reaction Force - QRF) à l'UNOSOM II. Le tableau dressé par la CE-ONU mentionne explicitement les actions conduites par la QRF et les Rangers; nous ferons de même. La somme des actions violentes entreprises par les forces de l'UNOSOM II s'élève à 40. Plusieurs de ces actions étant de nature multiple, comme dans le cas précédent, la somme des actions répertoriées dans notre tableau est plus élevée que 40.

\section{Tableau 2 : actions des forces de L'UNOSOM II contre la SNA}

\section{Arrestation 3}

Attaque aérienne 5

Attaque conjointe air-sol 1

Détenu(e) abattu(e) 1

Perquisition (recherche d'armes) 11

Raid (général) 7

Raid (QRF) 15

Raid (Rangers) 6

Tir d'artillerie (anti-mortier) 1

On constatera le nombre élevé des actions entreprises par la QRF et les troupes d'élite (Rangers) américaines. Un bon nombre de ces interventions des troupes d'élite américaines reposait sur un soutien aérien fourni par les hélicoptères de combat. Si l'on ajoute que plusieurs des actions américaines furent des opérations conjointes impliquant les contingents d'autres pays (le Bangladesh, l'Italie, la Malaisie et le Pakistan), on parvient à l'inévitable conclusion que les Etats-Unis ne se retirèrent jamais véritablement de la Somalie et que la puissance de feu de leurs armes y fut toujours aussi meurtrière. L'une des raisons du poids de la présence américaine tient au sous-équipement chronique des contingents en provenance de pays non industrialisés. Lorsqu'un état de guerre prévalut entre la SNA et les forces de l'UNOSOM II, seule la technologie américaine pouvait faire contrepoids à la faiblesse de plusieurs des forces de l'UNOSOM II ${ }^{26}$.

Pour brève qu'elle soit, cette analyse nous révèle à quel point l'application indistincte de concepts comme ceux de guerre et de violence sans égard aux formes concrètes qu'ils prennent sur le terrain ne peut produire que des analyses désincarnées et à la rigueur fallacieuses. L'épisode guerrier de l'intervention de l'ONU en Somalie est parcouru par un clivage si profond qu'il serait peut-être permis de parler de deux types 
de conflits qui se sont livrés de façon simultanée. Il y eut d'une part les attaques des forces de la SNA contre les troupes de l'ONU : ces attaques usaient la plupart du temps des moyens de la guérilla (les tirs de mortiers, les embuscades, les mines), bien que les troupes somaliennes puissent se révéler un adversaire redoutable dans un combat rangé, lorsqu'elles étaient forcées de s'y engager. Les tactiques utilisées par les forces de la SNA ont eues pour résultat un nombre relativement élevé d'incidents, concentrés dans des zones déterminées (par exemple, l'aéroport) et y faisant peu de victimes. Au niveau des contingents fournis par les nations non industrielles, la réplique des forces de l'ONU s'est située au même niveau que celui des attaques de la SNA, ces contingents étant parfois moins bien équipés encore que leurs adversaires. L'action des forces américaines s'est toutefois située à un niveau profondément différent, elle s'est caractérisée par l'introduction, au sein d'une guerre de fantassins dont l'armement était de qualité très variable, d'une partie de la panoplie des armes à la pointe de la technologie militaire. L'utilisation de ces armes sophistiquées ne constitue pas en soi un avantage suffisant pour emporter la décision sur le terrain: finalement, les Américains et les autres troupes de l'UNOSOM II se retireront, abandonnant le terrain à leurs adversaires somaliens. Si donc l'utilisation de ces armes n'assure pas la victoire, elle assure toutefois, comme nous le verrons en détail, que le prix que devra payer l'adversaire pour occuper le terrain sera très lourd.

Une seconde conclusion induite par l'examen des actions militaires en Somalie est le déficit massif des opérations sur le terrain par rapport à la doctrine d'intervention sécrétée dans les officines de l'ONU et par la myriade des centres d'études stratégiques qui lui sont rattachés. Autant les doctrines sont prolixes et foisonnent de distinctions entre le nombre croissant des variantes du maintien de la paix, autant elles se fondent dans l'indistinct lorsqu'elles sont plongées dans le creuset du recours à la violence. Toute la doctrine se réduit alors à la réaffirmation, parfois paradoxale, du principe du recours à la force armée en dernière instance. Cependant, une fois qu'on s'est résolu à franchir ce seuil, on perd tout sens de la mesure. Par exemple, les exigences de répondre de façon graduée à la violence s'effacent au profit d'une règle d'équivalence aveugle, selon laquelle un bombardement aérien " de précision " devient la mesure de rétorsion proportionnelle à un tir d'armes légères. Sous l'effet de sentiments qui oscillent entre la colère et la panique, on passe ainsi sans transition de l'autodéfense inquiète à l'agression sans remords ni retenue.

\section{NOTES}

1. CE-ONU, 1994, par. 201. Nous reprenons textuellement la terminologie de CE-ONU (1994), qui distingue entre " le pouvoir de désarmer " (the power to disarm) et " le pouvoir d'assister " (the power to assist). Nous ne discuterons pas l'épineuse question de savoir si l'expression " pouvoir d'assistance " n'est pas en elle-même contradictoire, la notion de pouvoir impliquant justement un débordement de la notion d'assistance au profit de celle de coercition et éventuellement d'imposition.

2. CE-ONU, 1994, par. 201, c'est nous qui traduisons. 
3. Pour une narration de ces travers de la politique américaine en Somalie, voir J. L. Hirsch et R. B. Oakley, op. cit., p. 124-139.

4. CE-ONU, 1994, par. 222.

5. Idem, par. 223.

6. Ibidem, par. 223.

7. Ibid, par. 224-225, c'est nous qui traduisons et soulignons.

8. Ibid, par. 270, c'est nous qui traduisons.

9. Ibid, par. 201- 211.

10. Ibid, par. 253.

11. Aux concepts précédemment distingués, on peut ajouter " l'édification d'une nation " (nationbuilding), la " reconstruction des Etats en faillite " (reconstruction of failed States) et le " multilatéralisme affirmatif " (assertive multilateralism). Voir à cet égard J. L. Hirsch et R. B. Oakley, op. cit., p. 151. Ce pullulement terminologique pédant s'explique en partie par le fait que les interventions de l'ONU dans des conflits se sont multipliées à partir de 1989. Il y a eu le même nombre d'opérations de maintien de la paix de l'ONU de 1989 à 1994 (15), que de 1945 à 1988 (15). Voir Jockel J.T., Canada and International Peacekeeping, Toronto, Canadian Institute of Strategic Studies, 1994. En 1989, le nombre des Casques bleus déployés s'élevait à 8000 ; il est aujourd'hui de plus de 80000 .

12. Cf. Hirsch J. L., Oakley R. B., op. cit., p. 151.

13. Segal D.R., Harris J. J., Rothberg J. M., Marlowe D. H., " Paratroopers as Peacekeepers ", Armed Forces and Society, Vol. 10, n 4, 1984, p. 487-506. Ajoutons que l'enquête de la COCANCIL a mis en lumière que le Régiment aéroporté était infiltré par des éléments appartenant à des groupes racistes notoires de l'extrême-droite (un parachutiste canadien s'est fait photographier en faisant le salut hitlérien; sa photo est parue dans le quotidien à plus grand tirage du Québec). Voir Kinsella W., Web of Hate, Toronto, Harpers Collins, 1994.

14. Worthless. Voir les transcriptions des audiences de la Commission d'enquête sur le groupement tactique du régiment aéroporté canadien/Board of Inquiry, Canadian Airborne Regiment Battle Group, 1993 (COCANMIL). Cette commission a publié un rapport qui résume ses travaux : Board of Inquiry, Canadian Airborne Regiment Battle Group, Report, Phase I, Vol. XI, Ottawa Department of National Defence.

15. Pour autant que nous ayons pu le constater à partir d'un examen documentaire, la situation fut la même pour le contingent belge.

16. Cf. COCANMIL, op. cit.

17. Purnelle M., op. cit, p. 124.

18. Cette terminologie francisée est le produit d'une traduction de l'américain, comme il est de coutume dans les Forces armées canadiennes. Elle n'est sans doute pas conforme à la terminologie usitée dans l'Armée française.

19. Cf. Hirsch J. L., Oakley R. B., op. cit., p. 27. à partir d'entrevues avec les dirigeants de l'UNOSOM I - Mohamed Sahnoun, James Jonah et David Bassiouni.

20. C'est nous qui traduisons à partir de la terminologie en usage dans les Forces armées canadiennes.

21. Dans le cas d'une menace posée par une ou des personnes non armées, on précise que la force meurtrière ne sera utilisée qu'en dernier recours. On précise aussi qu'on peut user d'agents de contrôle d'émeutes (par exemple, des gaz lacrymogènes), lorsqu'on dispose des autorisations nécessaires. La directive d'user de la force meurtrière contre une ou des personnes non armées, ne serait-ce qu'en dernier 
recours, est problématique : tirer dans une foule non armée - et a fortiori sur une personne sans arme - constituera toujours un geste controversé, même dans des cas extrêmes (pour empêcher, par exemple, le lynchage d'une personne par une foule en colère).

22. Fetherston A. B., Towards a theory of United Nations Peacekeeping, New York, St. Martin's Press, 1994.

23. Voir, à cet égard, les accablantes remarques de R. Brauman sur le spectacle donné par " les soldats de la paix ". Ces constatations sont confirmées par deux enquêtes canadiennes sur le comportement des troupes du Canada en Somalie. Brauman, op. cit., p. 26.

24. La liste compilée par la CE-ONU comprend des actions multiples sous un même titre; c'est pourquoi la somme des incidents que nous avons classifiés excède le chiffre de 140 .

25. RPG : Rocket Powered Grenade. Il s'agit d'un missile de faible puissance tiré à l'aide d'une arme similaire à un bazooka.

26. Il va sans dire que des contingents comme ceux de la Belgique, du Canada, de la France et de l'Italie ne souffraient pas des mêmes problèmes de sous-équipement pour les pays des nations non industrielles.

\section{INDEX}

Mots-clés : contrôle des foules, gestion de conflits, maintien de la paix, Militaires, organisations internationales

Index chronologique : 1990 - 2000

Thèmes : Nations Unies (ONU), US Army, Pentagone (US Department of Defence)

Index géographique : Corne de l'Afrique, Somalie 